\title{
Incidencia de Huesos Suturales en Cráneos de Changos del Norte de Chile, Región de Antofagasta
}

\author{
Incidence of sutural bones in a population of changos skulls of Northern Chile, Antofagasta. \\ "Fernando García-Hernández; **Juan Luis Díaz Blanco \& **Gabriel Thomas Murphy Echeverría
}

GARCÍA-HERNÁNDEZ, F; DÍAZ, B. J. L. \& MURPHY, E. G. T. Incidencia de huesos suturales en cráneos de changos del Norte de Chile, Región de Antofagasta. Int. J. Morphol., 25(4):801-804, 2007.

RESUMEN: La presencia de huesos suturales es producto de alteraciones en la formación de los huesos anchos del cráneo, considerándose también, variables étnicas, siendo de interés para la anatomía humana, antropología física, imagenología y medicina legal. Con la finalidad de contribuir al conocimiento de los huesos suturales y su incidencia en individuos originarios, se analizó su presencia en 23 cráneos de changos del Norte de Chile quienes se encuentran clasificados y pertenecen a la colección del Museo Regional de Antofagasta. Se comprobó la presencia de suturales en el 43,5\% de los casos, con un rango de 1 a 9, siendo bilaterales en el $60 \%$. Predominan los huesos en el lado izquierdo $(64,9 \%)$ y en cráneos braquicefálicos (IC=96,8). Se identificó el hueso lambda o interparietal en el $13,04 \%$ de los cráneos. Los datos obtenidos se corresponden con los descritos en la literatura.

PALABRAS CLAVE: Antropología Física; Cráneo; Huesos suturales.

\section{INTRODUCCIÓN}

Se cree que los huesos suturales, también conocidos como huesos wormianos, llamados así en virtud de la completa descripción que hizo, en 1611, el anatomista danés Olaüs Wormius o Worm (Sappey, 1888; Testut \& Latarjet, 1959), son producto de alteraciones en la formación de huesos anchos del cráneo a causa de alteraciones metabólicas del mesodermo, formándose generalmente a partir del tejido conectivo (Kahle et al., 1977), por centros de osificación anómalos (Orts Llorca, 1958), existiendo cierta correlación con la asimetría craneal, malformaciones de los huesos occipital y esfenoides, metopismo y anomalías congénitas del sistema nervioso central.

Los huesos suturales tienen importancia genética y hereditaria. Son considerados variables étnicas (Orts Llorca; Figún \& Garino, 1992), siendo de interés para la anatomía humana, antropología física, imagenología y medicina legal.

De acuerdo a la literatura, son estructuras variables en posición pese a que, con más frecuencia, se ubican a nivel de la articulación lambdoidea o parieto-occipital, situa- ción ya descrita por Sappey y reiterada por Sicher en 1965 y por otros autores; también es variable número, tamaño y forma, estando ubicados en suturas o fontículos.

Autores que han estudiado estas estructuras son entre otros: Sappey; Lips \& García-Hernández (1983); Henríquez-Pino et al. (1992) y Braga et al. (2000), entre otros.

El presente estudio pretende contribuir al conocimiento de los huesos suturales y su incidencia, en individuos originarios del Norte de Chile.

\section{MATERIAL Y MÉTODO}

Se analizaron 23 cráneos de changos del Norte de Chile, que habían sido recolectados en diversas áreas del litoral de la II Región y que pertenecen a la colección del Museo Regional de Antofagasta, Chile.

\footnotetext{
* Unidad de Anatomía, Departamento Biomédico, Facultad de Ciencias de la Salud, Universidad deAntofagasta, Chile.

** Ayudantes alumnos ad-honorem Asignatura de Anatomía Humana, Facultad de Ciencias de la Salud, Universidad de Antofagasta, Chile.
} 
Se dimensionaron la longitud anteroposterior máxima de cráneo y la anchura máxima de los cráneos.

Con estas mediciones se calculó el índice cefálico para determinar la forma del cráneo (braquicefalia, mesocefalia o dolicocefalia).

\section{RESULTADOS Y DISCUSIÓN}

Se comprobó presencia de huesos suturales, en 10 cráneos, lo que corresponde al $43,5 \%$ del total $(n=23)$ similar porcentaje al encontrado en cráneos brasileños por Henríquez-Pino et al. en 1992 (44,0\%). Figs. 1 a 4.

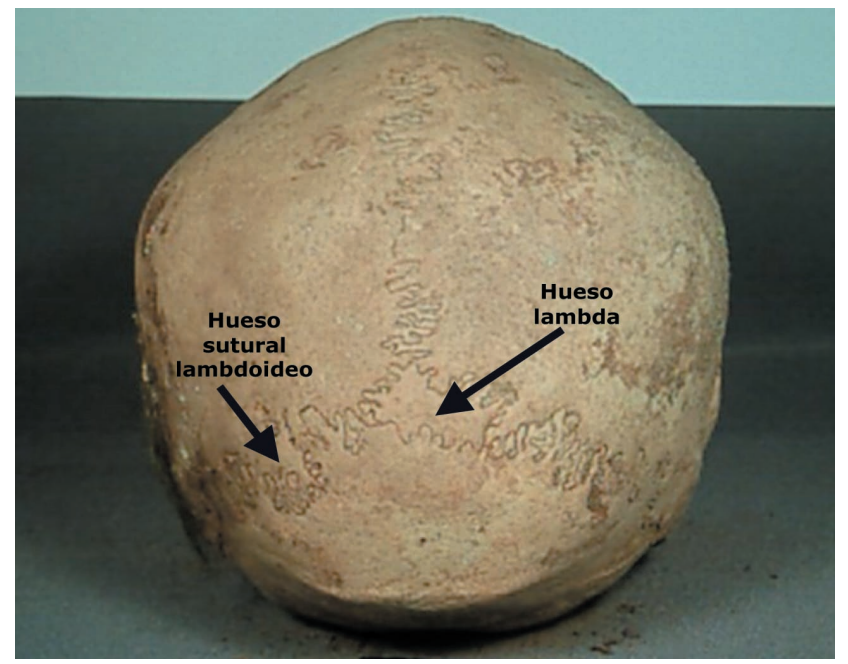

Fig. 1. Cráneo de un individuo chango del Norte de Chile, en norma posterior. Presencia de huesos sutural lambdoideo izquierda y lambda. Museo Regional de Antofagasta, Chile.

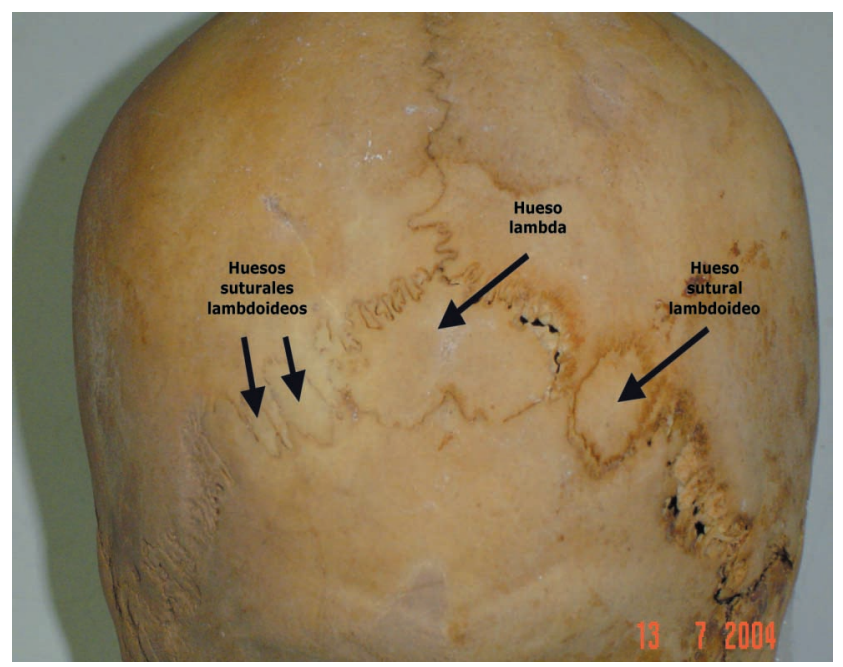

Fig. 2. Cráneo de un individuo chango del Norte de Chile, en norma posterior. Presencia de huesos suturales lambdoideos bilaterales y lambda. Museo Regional de Antofagasta, Chile.

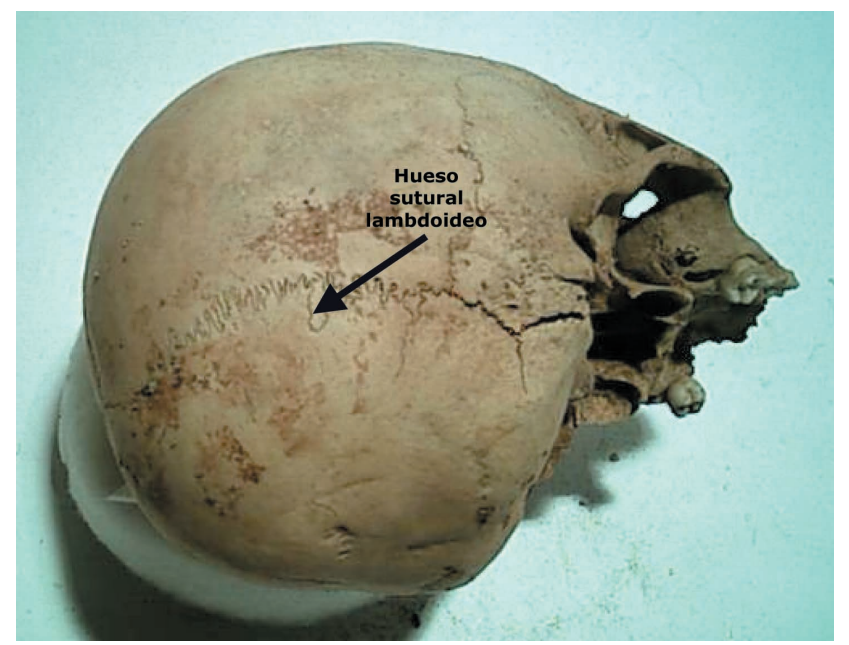

Fig. 3. Cráneo de un individuo chango del Norte de Chile, en norma lateral derecha. Presencia de hueso sutural lambdoideo derecho. Museo Regional de Antofagasta, Chile.

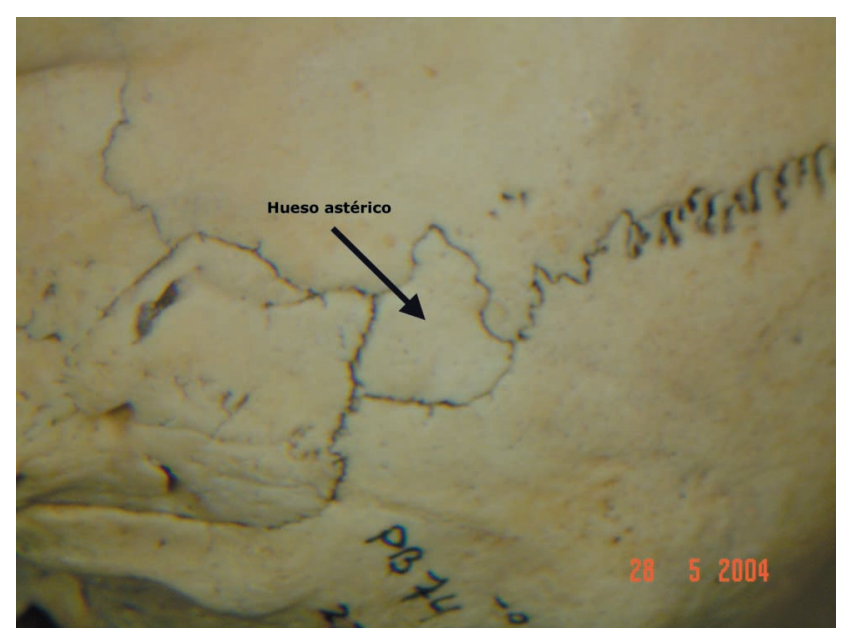

Fig. 4. Cráneo de un individuo chango del Norte de Chile, en norma lateral izquierda. Presencia de hueso sutural astérico. Museo Regional de Antofagasta, Chile.

El rango de frecuencia osciló entre 1 a 9 huesos suturales, en los 10 cráneos, siendo bilaterales en el $60 \%$ de los casos $(n=6)$. Predominaron los huesos suturales en el lado izquierdo $(64,9 \%)$, valor también coincidente con la investigación realizada por Henríquez-Pino et al. (42,5\%), aunque otros autores señalan un porcentaje mayor en el lado derecho.

Los huesos suturales predominaron en cráneos clasificados como braquicefálicos, lo que se corresponde con los antecedentes citados en la literatura por diversos autores (Orts Llorca; Figún \& Garino).

Se identificó el hueso lambda en $13,04 \%$ de los cráneos $(n=3)$, muy cercano al valor de $14,0 \%$ determinado también por Henríquez-Pino et al. 


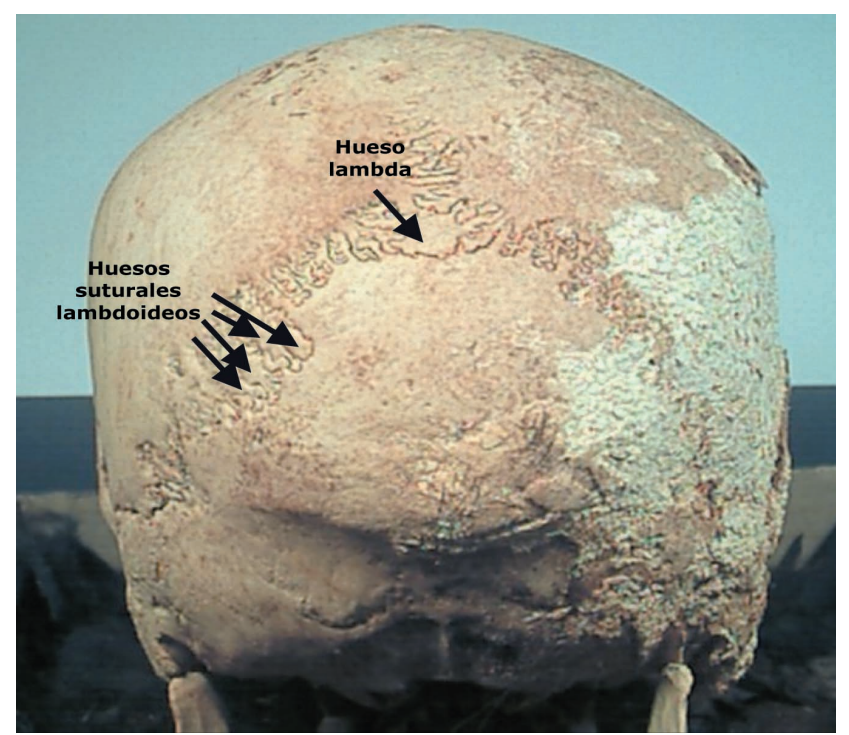

Fig. 5. Cráneo de un individuo chango del Norte de Chile, en norma posterior. Presencia de huesos suturales lambdoideos izquierdos y lambda. Museo Regional de Antofagasta, Chile.

No se identificaron en la muestra huesos suturales en la sutura coronaria, así como tampoco en bregma y en pterion.

Tabla I. Comparación de frecuencia porcentual de huesos suturales en diferentes muestras poblacionales.

\begin{tabular}{lc}
\hline \multicolumn{1}{c}{ POBLACIÓN } & $\%$ \\
\hline Chinos & 80,3 \\
Alemanes & 75,0 \\
Australianos & 72,6 \\
Edad hierro Romano Británica & 71,0 \\
Melanesios & 64,2 \\
Japoneses & 63,4 \\
Anglosajones & 55,6 \\
Hawaianos & 39,8 \\
Polinesios & 28,0 \\
Zona Norte de Chile (1983) & 54,6 \\
Zona Central de Chile (1983) & 36,3 \\
Cráneos indígenas (1983) & 84,6 \\
Brasileños (1992) & 44,0 \\
Chinos & 38,0 \\
Americanos no deformados & 39,0 \\
Boloñeses & 94,6 \\
Sardos (Cerdeña) & 88,6 \\
Changos (2005) & 43,5 \\
\hline
\end{tabular}

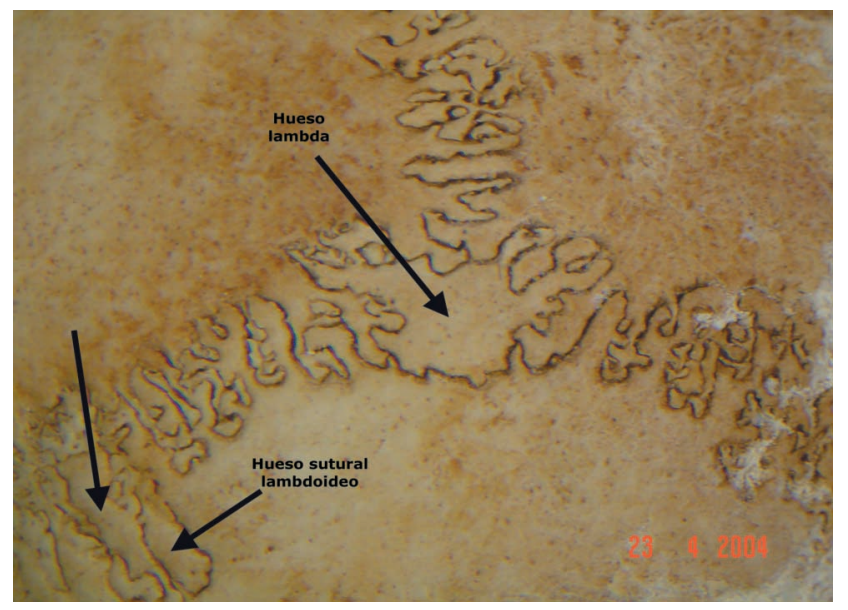

Fig. 6. Aumento de Fig. 5. para mostrar detalles de huesos sutulares lambdoideos izquierdos y lambda. Museo Regional de Antofagasta, Chile.

\section{CONCLUSIONES}

Los huesos suturales deben considerarse estructuras de presencia normal en cráneos humanos, en especial cuando se realizan diagnósticos en imagenología y en estudios de medicina legal.

Los huesos suturales se presentan en cráneos de diversos grupos poblacionales, no representando, por lo tanto, un indicador étnico. Además, los huesos suturales tienen variable ubicación, pudiendo ser uni o bilaterales, estar presentes en individuos de ambos sexos y en diferentes grupos étnicos.

GARCÍA-HERNÁNDEZ, F.; DÍAZ, B. J. L. \& MURPHY, E. G.T. Incidence of sutural bones in a population of chango's skulls of Northern Chile, Antofagasta Region. Int.J.Morphol., 25(4):801804, 2007.

SUMMARY: The presence of sutural bones is a product of alterations in the formation of the wide bones of the skull, in addition to ethnic variables, being of interest in human anatomy, physical anthropology, imagenology and legal medicine. With the purpose of contributing to the knowledge of the sutural bones and its incidence in original individuals, its presence in 23 skulls of changos of the North of Chile was analyzed that are classified and that they belong to the collection of the Regional Museum of Antofagasta. The presence of sutural bones in $43.5 \%$ of the cases with a rank from 1 to 9 was verified, being bilateral in $60 \%$. The bones in the left side $(64,9 \%)$ and in brachicephalic skulls predominate ( $\mathrm{IC}=96,8)$. The lambda or interparietal bone was identified in $13.04 \%$ of the skulls. The collected data correspond with the described ones by Literature.

KEY WORDS: Physical anthropology; Skulls; Sutural bones. 


\section{REFERENCIAS BIBLIOGRÁFICAS}

Braga, M. T. T.; Gabrielli, C. ; De SouzaA. ; Rodrigues, C.F.S. \& Marino, J.C. Sutural bones in the pterionRev. Chil.Anat. 18(1):97-101, 2000.

Figún, M. E. \& Garino, R. R. Anatomía Odontológica funcional y aplicada. $2^{\mathrm{a}}$ ed. Buenos Aires, El Ateneo, 1992.

Henríquez-Pino, J.; Buarque, L.C. \& Prates, J.C. Anatomical study of sutural bones in 200 Brazilian individuals skulls. Rev. Chil. Anat., 10(2):89-95, 1992.

Lips, W. \& García-Hernández, F. Estudios comparativos de la presencia de huesos wormianos en cráneos indígenas y su comparación con otras muestras poblacionales de Chile. Anal. Anat. Normal 1:95-99, 1983.

Kahle, W.; Leonhardt, H. \& Platzer, W. Atlas de Anatomía para estudiantes y médicos. $1^{\mathrm{a}}$ ed. Barcelona, Omega, 1977.

Orts-Llorca, F. Anatomía Humana. $2^{\mathrm{a}}$ ed. Barcelona, Editorial Científico Médico, 1958.

Sappey, PH. C. Traité d'Anatomie Descriptive. $4^{\mathrm{a}}$ ed. París, Adrien Delahaye et Émile Lecrosnier, Editeurs, 1888.

Sicher. Oral Anatomy. $4^{\text {a }}$ ed. Saint Louis, The C.V. Mosby Company, 1965.

Testut, L. \& Latarjet, A. Tratado de Anatomía Humana. $9^{a}$ ed. Barcelona, Salvat, 1959.
Dirección para correspondencia:

Prof. Dr. Fernando García-Hernández

Unidad de Anatomía

Departamento Biomédico

Facultad de Ciencias de la Salud

Universidad de Antofagasta

Antofagasta

CHILE

Email fgarcia@uantof.cl

Recibido : 22-12-2005

Aceptado: 17-04-2006 